\section{CLINICAL OUTCOMES OF TRANSFEMORAL VS TRANSAPICAL TAVI: UK SINGLE-CENTRE EARLY TAVI EXPERIENCE}

Mahvesh Javaid*, Nabeela Kareem, Simon Kennon, Wael Awad. Barts Health NHS Trust

\subsection{6/heartjnl-2017-311726.31}

Background Transcather Aortic Valve Replacement (TAVI) started at Barts Health NHS Trust (London Chest Hospital) in 2008. It was the beginning of a new era for patients with severe aortic stenosis who were not fit enough for surgery; giving another treatment opportunity to our patients.

Methods Data was collected on patients who underwent TAVI between January 2009-January 2015, comparing transapical (TA) and transfemoral (TF) approaches using appropriate statistical tests. The clinical outcomes included 30 day mortality, mean Log-EuroScore, renal function and complications.

Results Over the 6 years, there was a total of 79 TA $(39.1 \%)$ and 123 TF $(60.9 \%)$ TAVI procedures. 30 day mortality included TA $3.8 \%$ Vs TF 4.1\% ( $\mathrm{p}=0.213)$; with Mean LogEuroScore being 19.68 TA and 20.07 TF ( $p=0.879)$, which improved over the years. Mean hospital stay for TA was 13 days and TF was 11 days.

Patients who had an eGFR $<60$ and received a contrast volume $>150 \mathrm{ml}$ was $36.8 \%$ in TA patients and $35.8 \%$ in TF patients. With the former patient group, TA patients had a statistically significant post-TAVI AKI with worsening eGFR than TF $(p=0.0013)$. Also, these TA patients had a longer hospital admission (mean $=18.14$ days) compared to TF (mean=10.32 days). Those patients with eGFR $<60$ and received $<150 \mathrm{ml}$ contrast had similar outcomes in TA patients $(p=<0.0001)$ however there was a 1 mean day difference in hospital stay.

The complications during TAVI procedures included major bleeding (TA 6.33\% Vs TF 9.76\% p=0.081), arrhythmia/cardiac arrest (TA $3.80 \%$ Vs TF $0.81 \% \mathrm{p}=0.408$ ) and cardiac tamponade (TA 0\% Vs TF 4.07\% p=0.185). Other complications like para-prosthetic leak accounted for TA $8.47 \%$ Vs TF $14 \%(\mathrm{p}=0.700)$, and intra-operative regurgitation TA $11.43 \%$ Vs $21.28 \% \mathrm{TF}(\mathrm{p}=0.589)$.

Conclusions Both TA and TF approaches were observed to have similar clinical outcomes over those 6 years, except for those TA patients with poor renal function $(\mathrm{eGFR}<60)$, who face worsening post-TAVI AKI and requiring longer hospital stay for recovery; despite the contrast volume received. Overall, TAVIs have been successful at Barts Health since it began, therefore the number of cases is expanding every year.

\section{Cardiac Rhythm Management}

\section{GLOBAL HIGH DENSITY MAPPING OF RE-ENTRY VULNERABILITY INDEX AIDS RISK STRATIFICATION AND IDENTIFIES SITES OF ARRHYTHMIA INITIATION IN BRUGADA SYNDROME AND ARVC}

${ }^{1}$ Claire Martin* ${ }^{2}$ Michele Orini, ${ }^{2}$ Neil Srinivasan, ${ }^{2}$ Justine Bhar-Amato, ${ }^{1}$ Anthony Chow, ${ }^{1}$ Martin Lowe, ${ }^{1}$ Ron Simon, ${ }^{2}$ Peter Taggart, ${ }^{2}$ Pier Lambiase. ' Barts Heart Centre; ${ }^{2}$ University College London

\subsection{6/heartjnl-2017-311726.32}

Introduction Initiation of reentrant ventricular tachycardia (VT) involves complex interactions between activation (AT) and repolarization times (RT). Recently, an algorithm has been developed to generate a spatial map (designated the re-entry vulnerability index-RVI) from intervals between local ATs and RTs at adjacent points over a multielectrode grid. The algorithm has been shown to accurately identify the region of a macro-reentrant circuit in two animal models (1). The aim of this study was to test the feasibility of RVI mapping in the right ventricle (RV) to aid ablation strategy of reentrant arrhythmias.

Methods Patients with Arrhythmogenic Right Ventricular Cardiomyopathy (ARVC) $(\mathrm{n}=10)$, Brugada Syndrome $(\mathrm{BrS})$ $(n=14)$ and normal heart RV outflow tract (RVOT) VT $(n=6)$ underwent electrophysiological studies and were followed up for $113 \pm 21$ months post procedure. Unipolar electrograms were recorded from a non-contact array (St Jude Medical) placed in the RVOT and geometry created with the Ensite system. Recordings were made during a programmed electrical stimulation protocol consisting of a $600 \mathrm{~ms}$ drive train and S2 extras. ATs and RTs were computed from the unipolar electrograms and the RVI calculated as previously described (1). Minimum $10 \%$ of global RVI values corrected for cycle length $\left(R V I_{c}{ }^{10 \%}\right)$ and distance between region of lowest $5 \%$ of RVI values and region of earliest activation during VT $\left(D_{\text {min }}\right)$ were computed for each patient.

Results Shortest values of RVI, which represent sites of highest susceptibility to re-entry, co-localised with earliest activation point during VT in the 9 patients with ARVC/BrS where VT was initiated (figure 1). In the ARVC patients this corresponded with location of RV scar as seen on MRI. In the 6 patients with normal heart focal VT, regions of lowest RVI did not co-localise with VT origin. Distance between region of lowest RVI and region of earliest activation during VT, $D_{\text {min }}$,
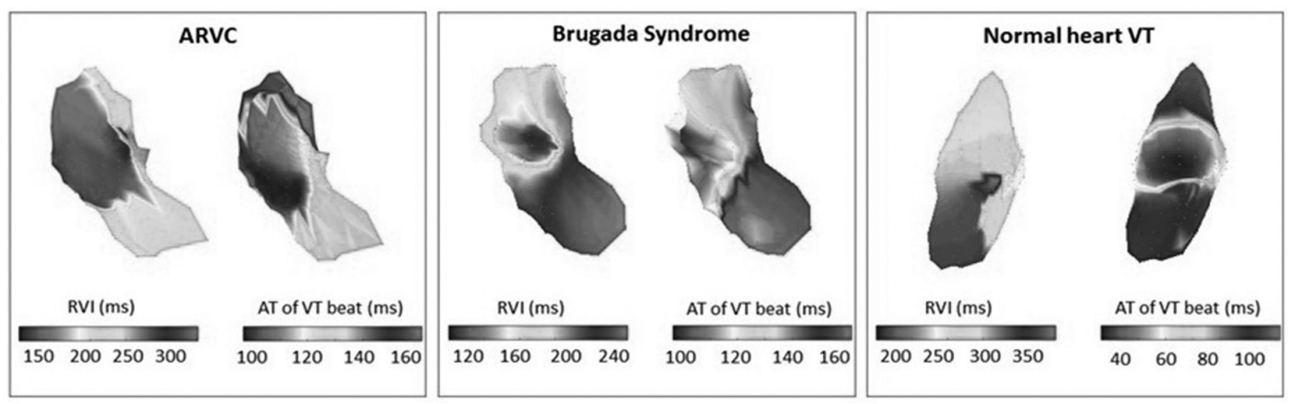

Abstract 32 Figure 1 